\title{
Peripheral Administration of Magnesium Sulfate and Ketamine Hydrochloride Produces Hypesthesia to Mechanical Stimuli in Humans
}

\author{
Osamu Iwatsu, ${ }^{*, a}$ Takahiro Ushida, ${ }^{a}$ Toshikazu Tani, ${ }^{a}$ Nada Bashir Lawand, ${ }^{b}$ \\ and Hiroshi Yamamoto ${ }^{a}$ \\ ${ }^{a}$ Department of Orthopedic Surgery, Kochi Medical School, Kohasu, Okoh-cho, Nankoku, Kochi 783-8505, Japan and ${ }^{b}$ Department \\ of Anatomy and Neuroscience, University Texas Medical Branch, 301 University Boulevard, Galveston, Texas, 77555-1069, U.S.A.
}

(Received October 9, 2001; Accepted October 22, 2001)

We investigated the functional significance of peripherally distributed glutamate, including $N$-methylD-aspartate (NMDA), receptors in humans. Magnesium sulfate (MG) and ketamine hydrochloride (KET), NMDA receptor antagonists, were injected into the skin of the medial region of forearm of healthy volunteers to investigate whether these substances are capable of changing the sensory transmission. We found that intracutaneous injections of MG and KET cause hypesthesia to noxious and innoxious mechanical stimulations. These results suggest that peripheral NMDA receptors are involved not only in sensory acceptance of noxious mechanical stimulation, but also in transmission of innoxious mechanical stimuli. The observation that the state of NMDA receptors in the skin may be affected by intracutaneous injection of their antagonists lays ground for possible clinical applications.

Key words $-N$-methyl-D-aspartate, magnesium sulfate, ketamine hydrochloride, hypesthesia

\section{INTRODUCTION}

$N$-methyl-D-aspartate (NMDA), a glutamic acid receptor, is localized in the central nervous system and was shown as responsible for the development of abnormal pain status, such as hyperalgesia and allodynia. In addition to their central location, recent studies identified NMDA receptors peripherally, including skin, ${ }^{1)}$ as well, and found that they play a role in the transmission of noxious signals. ${ }^{2)}$ In laboratory studies, systemic administration of

\footnotetext{
*To whom correspondence should be addressed: Department of Orthopedic Surgery, Kochi Medical School, Kohasu, Okohcho, Nankoku, Kochi 783-8505, Japan. Tel.: +81-88-880-2387; Fax: +81-88-880-2388; E-mail: hcb03073@nifty.com
}

noncompetitive antagonists of NMDA receptors, such as ketamine hydrochloride (KET) and MK-801, produced inhibition of pain responses and behaviors induced by peripheral administration of the agonists of glutamate receptor. ${ }^{3)}$ Using the formalin stimulation test, it was shown that pretreatment of local sites with MK-801 inhibited response to noxious stimulation. ${ }^{4)}$ It was also reported that administration of agonists of glutamic acid decreases the threshold for both noxious and innoxious stimulation. ${ }^{2,5)}$ These findings suggested that peripherally distributed NMDA receptors may be involved in the acceptance of noxious stimuli.

In previous experimental studies using rats, cutaneous injection of drugs was used as a noxious stimulation test. ${ }^{3,5)}$ However, animal experiments considerably limit the accurate estimation of the feeling of pain, since it is impossible to clarify whether the animals react to the pain caused by the specific injected drug or to the pain caused by the injection itself. Therefore, investigation of the sensory threshold using the von Frey filaments relies mainly on electrophysiological experiments. In the latter experiments, however, the animals are supposed to be anesthetized and the drug administered for the purpose of anesthesia may interfere with the response of other drugs with a general anesthetic effect, for instance KET. Taking all these limitations into account, we injected intracutaneously a small amount of low concentrated NMDA antagonists [magnesium sulfate (MG) and KET] into the skin of humans. Volunteers were asked to estimate the degree of pain, and the effects of these drugs on the peripheral NMDA receptors were examined. In addition to noxious mechanical stimulation, we performed an experiment evaluating the innoxious mechanical stimulation, such as tactile sensation, to investigate the possible involvement of NMDA receptors in the skin 
sensory acceptance of signals others than noxious stimuli.

\section{MATERIALS AND METHODS}

Twenty healthy volunteers who approved the study protocol were enrolled in the present experiment and underwent the procedure described below. Using the double-blind method, 10 men in each of 2 groups received intracutaneous injections of MG (age ranged from 26 to 34 years, mean: 29 years) and KET (age ranged from 26-36 years, mean: 30.8 years), respectively. $0.1 \mathrm{ml}$ of the drug solution was injected into the anterior ulnar site of forearm to form wheals: MG was used at concentrations of $0.5 \mathrm{M}$ and $0.05 \mathrm{M}$, osmotic pressure 3, $\mathrm{pH}$ 5.5-7.5; KET was used at concentrations of $1 \%(37 \mu \mathrm{M})$ and $0.1 \%(3.7 \mu \mathrm{M})$, osmotic pressure $1, \mathrm{pH} 3.5-5.5$. In controls, $0.1 \mathrm{ml}$ of physiological saline $(\mathrm{NaCl})$ was injected into the similar site of other forearm. Following injection, the produced effects were evaluated after 1, 10, 20,30, and $60 \mathrm{~min}$ for the MG site and after 1, 10, 20, and $30 \mathrm{~min}$ for the KET-injected site. The following tests were performed:

Tactile Sensation Evaluation — Using a horse hair brush, the tactile sensations of the wheal region formed by intracutaneous injection and the region of unaffected skin were compared.

Tactile Pressure Threshold Test — Using the von Frey filaments, the tactile pressure threshold was converted into numerical values.

Pinch-Pain Evaluation Test — Using an arterial clamp, pinch-evoked sense of pain of the wheal region formed by intracutaneous injection and unaffected normal proximal skin were compared. Rating the sense of pain of normal skin at the same arm as 10 , the sense of pain at the wheal region was evaluated using the tactile sensation pinch-pain evaluations. Accordingly, the differences between injected drug and $\mathrm{NaCl}$ were analyzed by Wilcoxson signed rank test and the repeated measures analysis of variance (ANOVA).

\section{RESULTS}

The tactile sensation caused by brush decreased by 1-20 min after injection of MG at concentration of 0.5 and $0.05 \mathrm{M}$ (Fig. 1-a) and by $1-10 \mathrm{~min}$ after injection of 1\% KET (Fig. 2-a) (repeated measures ANOVA: MG: $0.5 \mathrm{M}, p<0.0001 ; 0.05 \mathrm{M}$, $p<0.0001 ;$ KET: $1 \%, p<0.0001 ; 0.1 \%$, $p=0.9994)$, respectively. The tactile pressure threshold measured using the von Frey filaments increased by $1-20 \mathrm{~min}$ and by $1-10 \mathrm{~min}$ after injection of $0.5 \mathrm{M}$ and $0.05 \mathrm{M}$ of MG respectively (Fig. 1-b) and by 1-10 min after injection of either $1 \%$ or $0.1 \%$ KET (Fig. 2-b) (repeated measures ANOVA: MG: $0.5 \mathrm{M}$, $p<0.0001 ; 0.05 \mathrm{M}, p<0.05$; KET: $1 \%, p<0.01$; $0.1 \%, p<0.01$ ). In the cases of occurrence of hypesthesia and when it was difficult to determine the largest filament diameter, the value of the filament with largest diameter was regarded as the measured value, and the tactile pressure threshold was used as a reference. The pinch-pain evoked by an arterial clamp was similarly reduced by $1-10 \mathrm{~min}$ after injection of $0.5 \mathrm{M}$ and $0.05 \mathrm{M} \mathrm{MG}$ (Fig. 1-c) as well as $1 \%$ and $0.1 \%$ KET (Fig. 2-c) (repeated measures ANOVA: MG: $0.5 \mathrm{M}, p<0.0001 ; 0.05 \mathrm{M}$, $p<0.0001$; KET: $1 \%, p<0.0001 ; 0.1 \%$, $p<0.0001)$. In controls, intracutaneous injection of $\mathrm{NaCl}$ decreased the tactile sense and increased the sensory threshold measured using the von Frey filaments by no longer than 1 min after injection.

\section{DISCUSSION}

It is known that the action of central NMDA receptors in response to noxious stimulation includes formation of a secondary hyperalgesia zone. It was also shown that administration of NMDA receptor blockers, such as MG and KET, increases pain thresholds of a secondary hyperalgesia zone. ${ }^{6-8)} \mathrm{Al}-$ though it was reported that NMDA receptors are involved in the acceptance of noxious stimulation and can be blocked by their antagonists, ${ }^{2,4)}$ little attention has being paid so far to the functional importance of the peripherally distributed NMDA receptors. In the present study, we injected low concentrations of MG and KET into the human peripheral skin and observed hypesthesia for the primary noxious stimulation caused by an arterial clamp. The latter indicated that NMDA receptors on periphery are involved in the transmission of primary pain caused by noxious mechanical stimulation. In addition, we also observed hypesthesia for innoxiuous mechanical stimulation caused by brush and von Frey filaments.

Recent study of Ushida et $a .^{4)}$ evaluated the importance of mechanical stimulation by studying the relationship between magnesium ions and peripheral NMDA receptors. It was found that injec- 
Fig.1-a

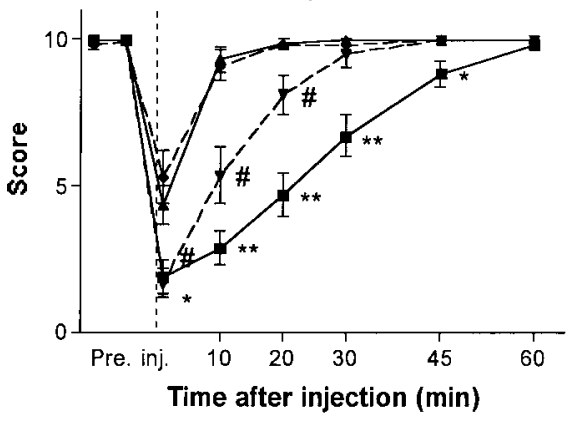

Fig.1-b

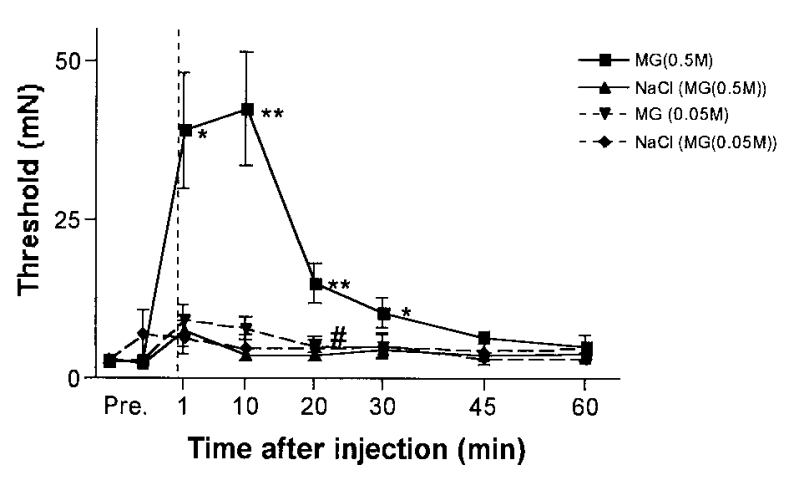

Fig. 1-c

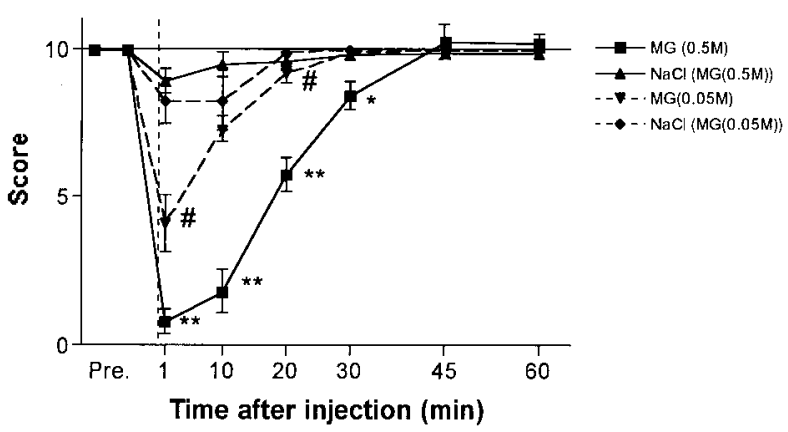

Fig. 1. Inhibitory Effect of Magnesium Sulfate on Mechanical Sensory Testing

Graphic representation of sensory thresholds at the injection site evaluated by brush (a), von Frey filaments (b) and a pinch (c) following administration of $0.05 \mathrm{M}$ MG. Note a significant increase of the sensory threshold using brush, von Frey filaments and a pinch. (0.5 M of MG significantly different from $\mathrm{NaCl}(0.5 \mathrm{M}$ of $\mathrm{MG})$. (Wilcoxson signed rank test, $\left.{ }^{*} p<0.05,{ }^{* *} p<0.01\right), 0.05 \mathrm{M}$ of MG significantly different from $\mathrm{NaCl}(0.05 \mathrm{M}$ of $\mathrm{MG})$.(Wilcoxson signed rank test, $\# p<0.05$, $\# \# p<0.01))$

tion of MK-801, a NMDA receptor antagonist, into the peripheral skin of rats produces inhibition of innoxious stimulation. Since MK-801 is known to be able to non-competitively antagonize the central NMDA receptors via magnesium ions, it seems likely that administration of magnesium ions produced inhibition of NMDA receptors distributed in skin. In agreement with these results, KET caused hypes-
Fig.2-a

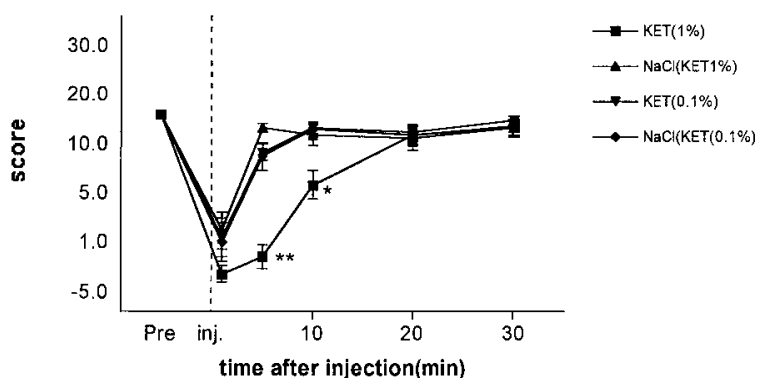

Fig.2-b

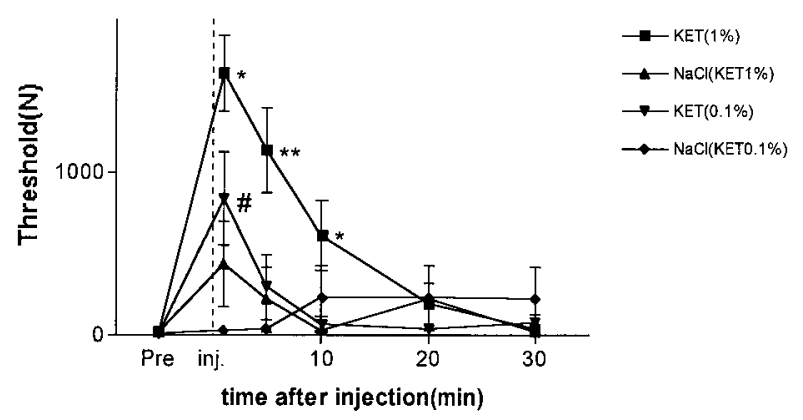

Fig.2-c

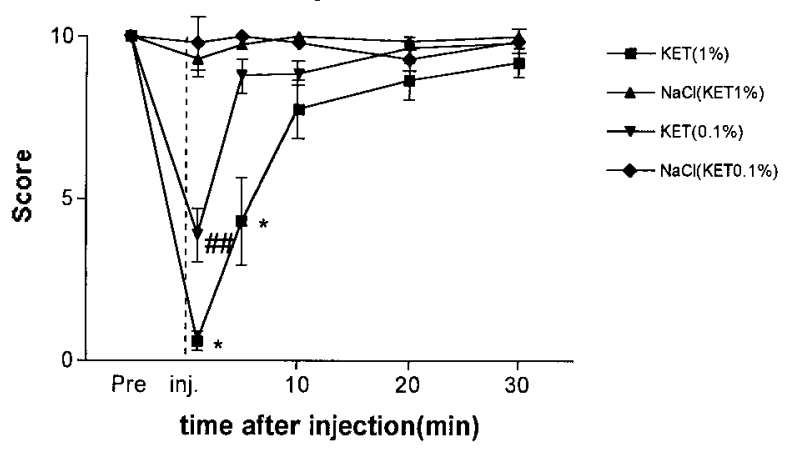

Fig. 2. Inhibitory Effect of Ketamine Hydrochloride on Mechanical Sensory Testing

Graphic representation of sensory thresholds at the injection site evaluated by brush (a), von Frey filaments (b) and a pinch (c) following administration of KET at concentrations of $1 \%$ and $0.1 \%$. A significant increase of the sensory threshold is noticed ( $1 \%$ of KET significantly different from $\mathrm{NaCl}\left(1 \%\right.$ of KET). (Wilcoxson signed rank test, ${ }^{*} p<0.05$, $* * p<0.01), 0.1 \%$ of KET significantly different from $\mathrm{NaCl}(0.1 \%$ of KET). (Wilcoxson signed rank test, $\# p<0.05, \# \# p<0.01$ ))

thesia for innoxious mechanical stimulation in our study. It should be mentioned that KET injected into avascularized vein in upper extremities induces local anesthesia at the concentration of $0.3 \%$ and higher. ${ }^{9)}$ In our study, hypesthesia was observed at the concentration of $0.1 \%$. Since the concentration used by us is considerably lower than the one required for anesthetic purposes, it seems unlikely that the local anesthetic effects were of significance in the present study. Therefore, we can conclude that peripheral NMDA receptors may play a role in the 
transmission of innoxious stimulation, such as tactile sensation to the central nervous system. Importantly, immunocytochemical studies reported that the number of NMDA receptors on peripheral nerve fibers increases in chronic inflammation. ${ }^{10)}$ It is possible that inflammation stimulates peripheral NMDA receptors and eventually increases irritability of nerve fibers. In clinical settings, patients with causalgia experience severe pain due innoxious stimulation. It is also known that intracutaneous administration of KET inhibits hyperalgesia after burning. $\left.{ }^{6}\right)$ Since KET and MG are commonly used as a general anesthetic agent and a therapeutic drug for hypomagnesaemia and eclampsia respectively, peripheral NMDA receptor-blocking effect of these drugs may be of clinical relevance.

In conclusion, results of the present for the first time show that NMDA receptors distributed on periphery may be involved not only in acceptance of noxious stimulation, but also in sensory transmission of innoxious mechanical stimulation. The observation that intracutaneous injection of NMDA receptor antagonists can influence the state of receptors lays ground for their possible clinical use.

\section{REFERENCES}

1) Carlton, S. M., Hargett, G. L. and Coggeshall, R. E. (1995) Localization and activation of glutamate receptors in unmyelinated axons of rat glabrous skin. Neurosci. Lett., 197, 25-28.

2) Zhou, S., Bonasera, L. and Carlton, S. M. (1996) Peripheral administration of NMDA, AMPA or KA results in pain behaviors in rats. Neuroreport, 7, 895900.

3) Davidson, E. M. and Carlton, S. M. (1998) Intraplantar injection of dextrorphan, ketamine or memantine attenuates formalin-induced behaviors. Brain Res., 785, 136-142.

4) Ushida, T., Tani, T., Kawasaki, M., Iwatsu, O. and Yamamoto, H. (1999) Peripheral administration of an N-methyl-D-aspartate receptor antagonist (MK801 ) changes dorsal horn neuronal responses in rats. Neurosci. Lett., 260, 89-92.

5) Davidson, E. M., Coggeshall, R. E. and Carlton, S. M. (1997) Peripheral NMDA and non-NMDA glutamate receptors contribute to nociceptive behaviors in the rat formalin test. Neuroreport, $\mathbf{8}$, 941-946.

6) Warncke, T., Jorum, E. and Stubhaug, A. (1997) Local treatment with the N-methyl-D-aspartate receptor antagonist ketamine, inhibit development of secondary hyperalgesia in man by a peripheral action. Neurosci. Lett., 227, 1-4.

7) Takano, Y., Sato, E., Kaneko, T. and Sato, I. (2000) Antihyperalgesic effects of intrathecally administered magnesium sulfate in rats. Pain, 84, 175-179.

8) Ishizaki, K., Sasaki, M., Karasawa, S., Obata, H., Nara, T. and Goto, F. (1999) The effect of intrathecal magnesium sulphate on nociception in rat acute pain models. Anaesthesia, 54, 241-246.

9) Durrani, Z., Winnie, A. P., Zsigmond, E. K. and Burnett, M. L. (1989) Ketamine for intravenous regional anesthesia. Anesth. Analg., 68, 328-332.

10) Carlton, S. M. and Coggeshall, R. E. (1999) Inflammation-induced changes in peripheral glutamate receptor populations. Brain Res., 820, 6370 . 